\title{
Plasmonic Lenses for Ultrafast Electron Nanoemission
}

\author{
Daniel B. Durham ${ }^{1,2}$, Fabrizio Riminucci ${ }^{3,4}$, Kostas Kanellopulos ${ }^{4,5}$, Silvia Rotta Loria ${ }^{4,6}$, Filippo \\ Ciabattini $^{7}$, Andrea Mostacci ${ }^{8}$, Andrew M. Minor ${ }^{1,2}$, Stefano Cabrini ${ }^{4}$, and Daniele Filippetto ${ }^{9}$ \\ ${ }^{1}$ Department of Materials Science and Engineering, University of California, Berkeley, Berkeley, California 94720, USA \\ ${ }^{2}$ National Center for Electron Microscopy, Molecular Foundry, Lawrence Berkeley National Laboratory, One Cyclotron Road, Berkeley, \\ California, 94720, USA \\ ${ }^{3}$ Department of Mathematics and Physics, University of Salento, Campus Ecotekne, 73100 Lecce, Italy \\ ${ }^{4}$ Molecular Foundry, Lawrence Berkeley National Laboratory, One Cyclotron Road, Berkeley, California, 94720, USA \\ ${ }^{5}$ Polytechnic University of Turin, Corso Duca degli Abruzzi, 24, 10129 Torino, Italy \\ ${ }^{6}$ Polytechnic University of Milan, Piazza Leonardo da Vinci, 32, 20133 Milano, Italy \\ ${ }^{7}$ Department of Information Technology and Electrical Engineering, ETH-Zurich, CH-8092 Zurich, Switzerland \\ ${ }^{8}$ University of Rome "La Sapienza", Piazzale Aldo Moro 5, 00185 Rome, Italy \\ ${ }^{9}$ Accelerator Technology and Applied Physics Division, Lawrence Berkeley National Laboratory, One Cyclotron Road, Berkeley, California, \\ 94720, USA \\ dbdurham@berkeley.edu
}

\begin{abstract}
We show the capability of plasmonic lenses for next-generation ultrafast electron sources. Using electromagnetic simulations, we design structures capable of femtosecond, nanoscale electron pulses. Plasmonic properties of template-stripped gold prototypes are characterized using cathodoluminescence spectromicroscopy. @ 2020 The Author(s)
\end{abstract}

As ultrafast science moves towards more nanoscale and heterogeneous systems, bright sources of femtosecond and nanoscale electron pulses are in demand [1]. While capable tip emitters have been demonstrated [2], they are limited in some experimental parameters; for instance, they provide few electrons per pulse and are susceptible to breakdown under high extraction fields like those used in radiofrequency MeV electron guns. Recently, nanostructured metallic cathodes have been demonstrated which exploit surface plasmons to enhance and localize the optical fields from the incident laser pulse at the surface $[3,4]$. While this approach has mainly been used to improve photocathode efficiency, the spatial and temporal characteristics of the emission can also be tailored by informed design of the nanostructures. Here, we show that plasmonic lenses enable femtosecond, nanoscale electron emission from flat metallic cathodes, showing promise as next-generation photoemitters for ultrafast nanoscience.
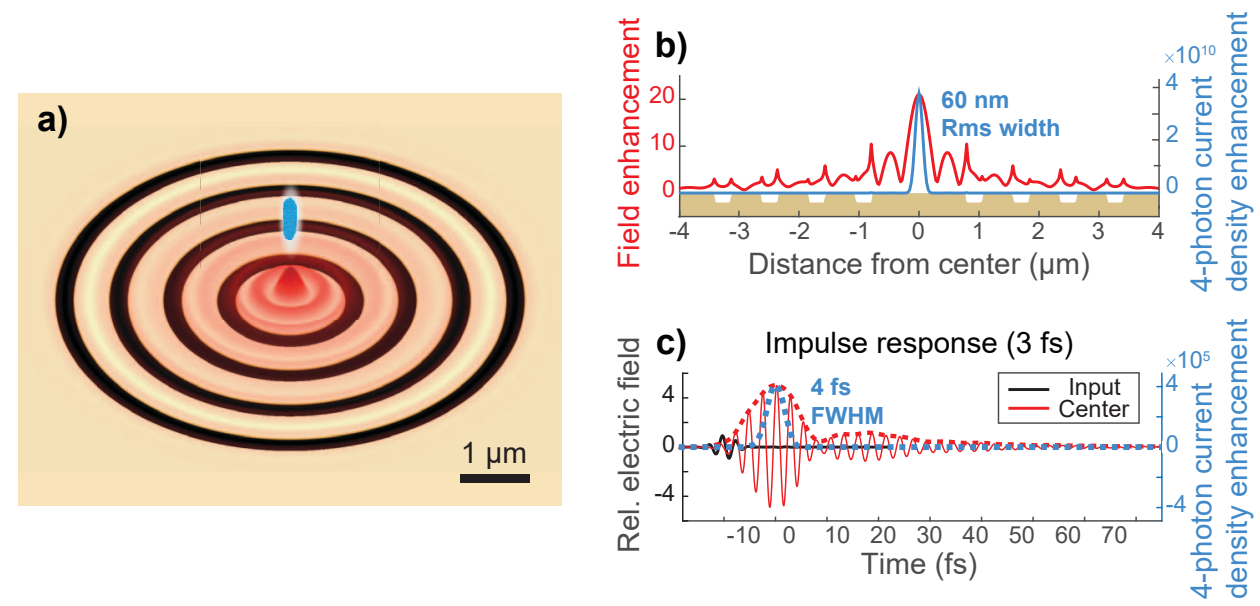

Fig. 1. Design and simulation of plasmonic lens emitter. (a) Illustration of concept and geometry. Simulated electric field distribution is shown in red as well as a cartoon emitted electron bunch in blue. Grooves (black) are $90 \mathrm{~nm}$ deep and $270 \mathrm{~nm}$ wide with $783 \mathrm{~nm}$ period. (b) Simulated electric field profile using a radially polarized $800 \mathrm{~nm}$ wavelength laser using 0.07 numerical aperture. The four-photon current density is superimposed, computed as eighth power of the field. (c) Simulated impulse response given relative to the maximum input field using a 3-fs FWHM laser pulse.

Bullseye plasmonic lenses couple radially polarized light into surface plasmon polaritons (SPPs) that are focused to maximum intensity at the structure center. Using Lumerical finite-difference time-domain (FDTD) simulations, we find that a four-ring plasmonic lens patterned into a gold surface can produce sub-100 nm RMS and sub-10 fs FWHM photoelectron pulses from 800-nm wavelength laser pulses [5] (see Figure 1). This design uses four-photon photoemission, taking advantage of the scaling of emission as the fourth power of optical intensity to 
suppress edges and tails and produce a dominant single electron bunch in space and time. Furthermore, this emission is generated from a flat surface rather than from edges or tips, which could reduce emittance and aberrations of the emitted pulses. While just one configuation is shown here, the pulse characteristics are widely adaptable by tuning the structure geometry: For instance, longer pulses with higher photocurrent can be generated by using more rings in the bullseye grating [5].
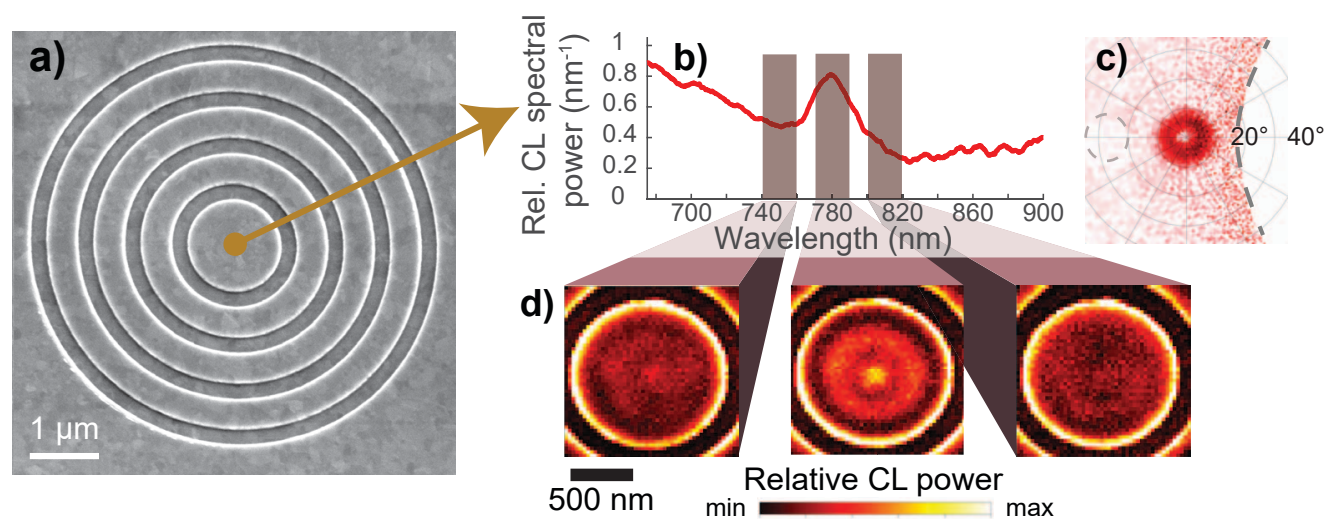

Fig. 2. Prototyping and characterization of plasmonic lens emitters. (a) SEM image of a structure produced by e-beam lithography and template stripping. (b) Relative (Rel.) cathodoluminescence spectrum when the electron beam is at the center. (c) Measured far field distribution using a 40-nm wide bandpass filter centered at $800 \mathrm{~nm}$ while the electron beam is at the center. Dashed lines indicate edges of parabolic collection mirror. (d) CL maps as function of electron beam position for the three wavelength bands highlighted in (b).

Using e-beam lithography and template stripping, we fabricated high quality prototypes in gold films with subnm surface roughness [5] (Figure 2a). To study the interaction of SPPs with the structure, we performed cathodoluminescence (CL) spectromicroscopy using a modified scanning electron microscope [5, 6] (see Figure 2b-d). When the electron beam is focused at the center, a broadband distribution of SPPs is generated and propagates radially outward to the bullseye grating, coupling out to a broad optical emission spectrum. Notably, there is a peak in emission near $800 \mathrm{~nm}$ photon wavelength corresponding to an expected resonance mode in the central plateau, which serves to further enhance the optical field at the center. This mode is spatially visualized when mapping the CL intensity as a function of electron beam position, and it has a similar central lobe diameter as the FDTD simulation. Also, the emitted light around $800 \mathrm{~nm}$ wavelength has a donut-shaped intensity distribution in the far field, showing good circular symmetry of the structure and that radial SPPs outcouple effectively to radially polarized light.

Ongoing work aims to demonstrate and characterize photoemission from such plasmonic lens cathodes. If realized, these structures could drive significant advances in ultrafast electron instrumentation and enable a variety of studies including higher-throughput spatiotemporal mapping and studies of nanoscale objects with $\mathrm{MeV}$ electrons. This work motivates further exploration of plasmonics for engineering the spatial and temporal characteristics of electron beams.

\section{References}

1. F. Ji, D. B. Durham, A. M. Minor, P. Musumeci, J. G. Navarro, and D. Filippetto, "Ultrafast relativistic electron nanoprobes," Communications Physics, vol. 2, no. 1, p. 54, 2019.

2. R. Bormann, S. Strauch, S. Schäfer, and C. Ropers, "An ultrafast electron microscope gun driven by two-photon photoemission from a nanotip cathode," Journal of Applied Physics, vol. 118, no. 17, p. 173105, 2015.

3. A. Polyakov, C. Senft, K. F. Thompson, J. Feng, S. Cabrini, P. J. Schuck, H. A. Padmore, S. J. Peppernick, and W. P. Hess, "Plasmon-enhanced photocathode for high brightness and high repetition rate x-ray sources," Physical Review Letters, vol. 110, no. 7, p. 076802, 2013.

4. R. K. Li, H. To, G. Andonian, J. Feng, A. Polyakov, C. M. Scoby, K. Thompson, W. Wan, H. A. Padmore, and P. Musumeci, "Surface-plasmon resonance-enhanced multiphoton emission of high-brightness electron beams from a nanostructured copper cathode," Physical Review Letters, vol. 110, no. 7, p. 074801, 2013.

5. D. B. Durham, F. Riminucci, F. Ciabattini, A. Mostacci, A. M. Minor, S. Cabrini, and D. Filippetto, "Plasmonic lenses for tunable ultrafast electron emitters at the nanoscale," Physical Review Applied, vol. 12, no. 5, p. 054057, 2019.

6. D. B. Durham, D. F. Ogletree, and E. S. Barnard, "Scanning Auger spectromicroscopy using the ScopeFoundry software platform," Surface and Interface Analysis, vol. 50, no. 11, pp. 1174-1179, 2018. 